\title{
Hepatomesenteric trunk, gastrosplenic trunk, coiled splenic and hepatic arteries, and a variant of Bühler's arc
}

\author{
M. Şelaru1*, M.C. Rusu2*, A.M. Jianu*(D), F. Bîrsășteanu**, B.A. Manta ${ }^{5 *}$ \\ 'Department of Surgery, Faculty of Medicine, "Victor Babes" University of Medicine and Pharmacy, \\ Timişoara, Romania \\ 2Division of Anatomy, Faculty of Dental Medicine, "Carol Davila" University of Medicine and Pharmacy, \\ Bucharest, Romania \\ ${ }^{3}$ Department of Anatomy and Embryology, Faculty of Medicine, "Victor Babes" University of Medicine \\ and Pharmacy, Timisoara, Romania \\ ${ }^{4}$ Department of Radiology and Medical Imaging, Faculty of Medicine, "Victor Babes" University of Medicine \\ and Pharmacy, Timisoara, Romania \\ 5"Carol Davila" University of Medicine and Pharmacy, Bucharest, Romania
}

[Received: 19 August 2020; Accepted: 23 October 2020; Early publication date: 3 November 2020]

The coeliac trunk is normally divided into the left gastric artery (LGA), splenic artery, and common hepatic artery (CHA). The combination between these arteries and the superior mesenteric artery (SMA) generates various combinations. We report here such a rare anatomic variant, namely the hepatomesenteric trunk (HMT), combined with a gastrosplenic trunk (GST). The variant was identified using computed tomography angiograms of a 62-year-old woman. The GST emerged from the aorta within the aortic hiatus of the diaphragm, a previously unknown possibility. Further, an accessory left hepatic artery originated from the LGA. The phrenic arteries had independent aortic origins. The HMT divided into the CHA and the SMA posterior to the origin of the hepatic portal vein (PV), above the pancreas. The CHA initially had a right course, towards the superior border of the $P V$, then it descended with a transpancreatic course posterior to the $P V$, reached its inferior/right border, and divided antero-inferiorly to the $P V$ into the proper hepatic and gastroduodenal arteries. The proper hepatic artery continued on the anterior side of the $P V$, sending off the left and right hepatic arteries. The HMT and the GST were connected by a rudimentary variant of the arc of Bühler, unreported previously. Arterial variations in the coeliac region are accurately distinguished on computed tomography angiograms. They should be evaluated by surgeons when different surgical procedures are evaluated. (Folia Morphol 2021; 80, 4: 1032-1036)

Key words: aorta, coeliac trunk, superior mesenteric artery, computed tomography, hepatic artery 


\section{INTRODUCTION}

The coeliac trunk (CT) normally sends off the left gastric artery (LGA), splenic artery (SA) and common hepatic artery (CHA) [6, 26].

The presence of aberrant hepatic arterial anatomy increases the surgical complexity and subsequently the potential risk of injury to the hepatic arterial supply during pancreaticobiliary procedures and duodenopancreatectomies [24]. The aortic common origin of the CHA and superior mesenteric artery (SMA) is by a hepatomesenteric trunk (HMT) $[9,15,16,21,22]$. This is a rare anatomic variation $[21,24]$. The HMT was encountered in $0.5 \%$ [1], 2\% [5], 2.08\% [15], and in $4.47 \%$ [33]. Just a couple of studies found the HMT by computed tomographic studies [13, 33]. There are two morphological possibilities when a HMT is formed: either the LGA and SA have independent aortic origins, or they have a common aortic origin - the gastrosplenic trunk (GST) [33].

We report here a HMT, characterised anatomically on computed tomography angiograms, with unusual topography of the co-existing GST, and a rudimentary arc of Bühler uniting the two trunks.

\section{MATERIALS AND METHODS}

The anatomic variants reported here were found during a retrospective study of computer tomography scans, in a 62-year-old woman. Briefly, the study consisted in injecting an iodine radiocontrast agent in the left brachial vein $(100 \mathrm{~mL}$, with $6 \mathrm{~mL} / \mathrm{s}$ flow), followed by $50 \mathrm{~mL}$ iodine radiocontrast agent (Ultravist $370 \mathrm{mg} / \mathrm{mL}$ ) in the brachial vein, and by $20 \mathrm{~mL}$ saline medium. The computed tomography was performed with a 32-slice scanner (Siemens Multislice Perspective Scanner), using a $0.6 \mathrm{~mm}$ collimation and reconstruction of $0.75 \mathrm{~mm}$ thickness with $50 \%$ overlap for multiplanar and three-dimensional (3D) volume rendering technique [26]. The arterial variant was documented using the Horos software and its 3D Volume Rendering application.

\section{RESULTS}

The abdominal aorta (AA) coursed retroperitoneally and was deviated to the right at the level of the third and fourth lumbar vertebrae. The aortic bifurcation into the common iliac arteries was at the level of the fifth lumbar vertebra.

A gastrosplenic trunk (GST) emerged on the anterior surface of the AA at $1.67 \mathrm{~cm}$ above the origin of the HMT. The GST runoff was from the anterior

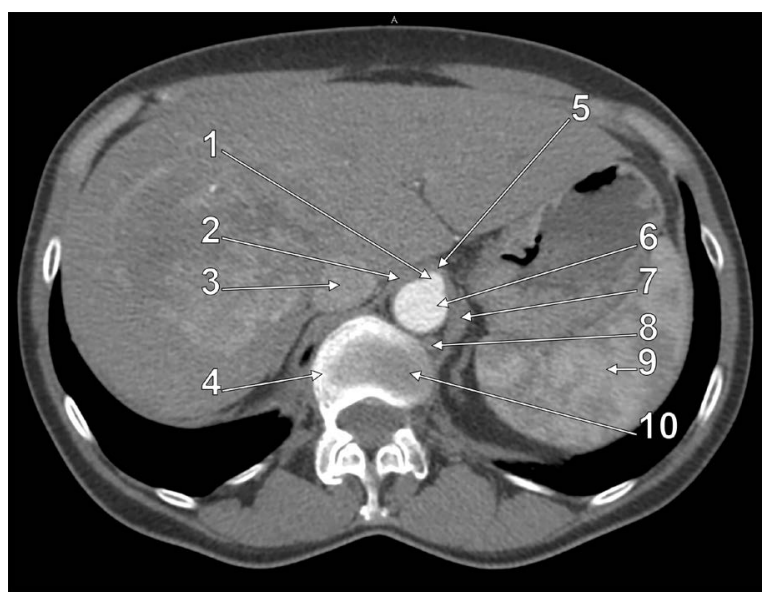

Figure 1. Axial coeliac trunk slice through the origin of the coeliac trunk which is located within the aortic hiatus of the diaphragm; 1 - coeliac trunk; 2 - right pillar of the diaphragm; 3 - inferior cava vein; 4 - first lumbar vertebra; 5 - median arcuate ligament; 6 - descending aorta; 7 - left pillar of the diaphragm; 8 - hemiazygos vein; 9 - spleen; 10 — intervertebral disc between T12 and L1.

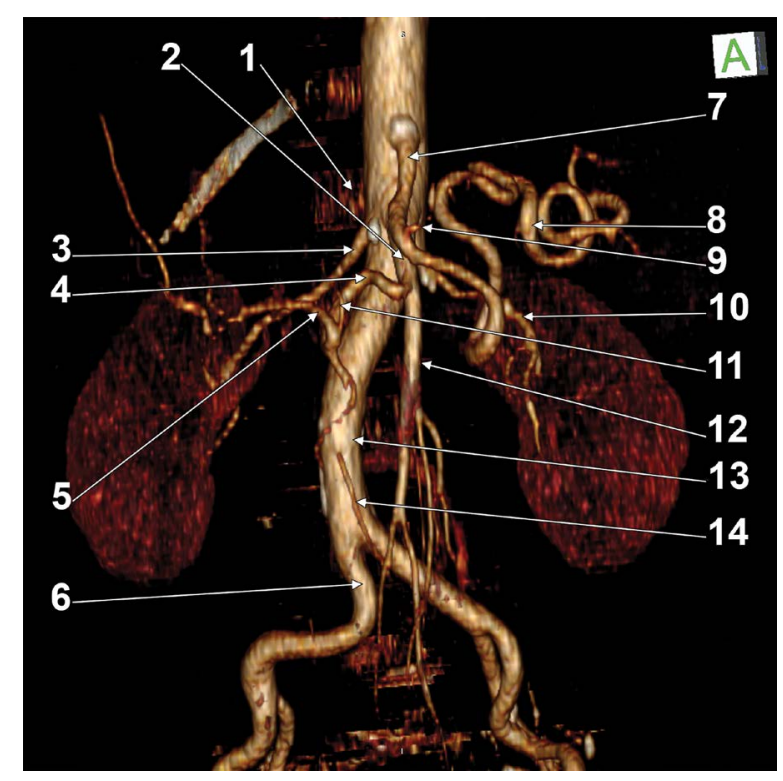

Figure 2. Three-dimensional volume renderisation of the abdominal aorta and its branches; 1 - first lumbar vertebra; 2 - hepatomesenteric trunk; 3 - right renal artery; 4 - common hepatic artery; 5 - right hepatic artery; 6 — right common iliac artery; 7 - gastrosplenic trunk; 8 - splenic artery; 9 - left gastric artery; $10-$ left renal artery; 11 - left hepatic artery; 12 - superior mesenteric artery; 13 - abdominal aorta; 14 — inferior mesenteric artery.

midportion of the aorta. The origin of the GST was within, and not inferior to the aortic hiatus of diaphragm, at the level of the intervertebral disc between T12 and L1 (Fig. 1). Just above the origin of the GST was identified an atheromatous plaque of the anterior aortic wall. The GST further branched the LGA and continued with the SA (Fig. 2). The later had a kinked 
and coiled morphology (Fig. 2). We could not find a dorsal pancreatic artery originating from the SA.

The two inferior phrenic arteries, right and left, originated independently from the posterior surface of the AA above the level of the origin of the GST.

The inferior mesenteric vein (IMV) extended toward the posterior left side of the SMA, circled the right side of the SMA, and united anteriorly with the superior mesenteric and splenic veins. It thus resulted the hepatic portal vein (PV) which inclined right abruptly towards the hilum of the liver (Fig. 3).

The AA was crossed anteriorly by the left renal vein at the level of the second lumbar vertebra. The HMT divided into the CHA and the SMA anterior to the left renal vein and posterior to the origin of the PV, above the pancreas. The initial segment of the CHA was directed to the right towards the superior border of the PV, then it descended with a transpancreatic course posterior to the PV, reached its inferior/right border, and divided antero-inferiorly to the PV into the proper hepatic (PHA) and gastroduodenal (GDA) arteries. The PHA continued on the anterior side of the $\mathrm{PV}$, which was thus contained within an arterial coil of the CHA and PHA. From the initial segment of the PHA left a rudimentary left hepatic artery (LHA), the left lobe of the liver being also supplied by an accessory LHA emerging from the LGA. Then, the PHA continued as right hepatic artery (RHA) and was further divided in anterior and posterior branches (Fig. 2).

The aortic origins of the HMT and of the right renal artery were at the level of the inferior border of the first lumbar vertebra. The left renal artery emerged the abdominal aorta at the level of the superior border of the second lumbar vertebra.

The HMT and the GST had comparable lengths (Table 1), and were connected by a variant of the arc of Bühler (Fig. 4).

\section{DISCUSSION}

A combination of GST and HMT, such as in this case, was found in $1.1 \%$ [20], thus being rare. In one of the cases described in the scientific literature, the left phrenic artery branched from the GST, but in the other two the GST origin of a phrenic artery was not found [20]. Similar to our case, the phrenic arteries arose independently from the aorta. Nakamura et al. (2003) [20] documented different studies regarding the prevalence of the GST-HMT variant and found it varying from $0.4 \%$ to $1.6 \%$. A recent multidetector computed tomography study on 1569 cases found

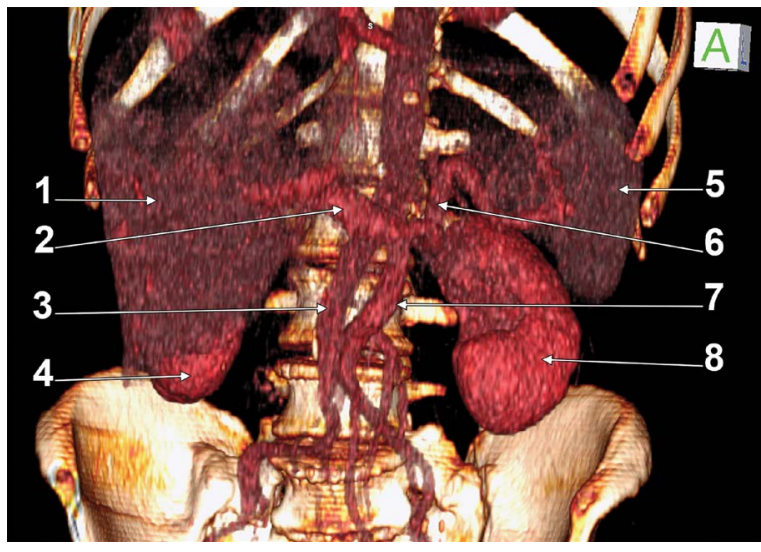

Figure 3. Three-dimensional volume renderisation of the hepatic portal venous system; 1 - liver (hepatomegaly); 2 - portal vein; 3 - superior mesenteric vein; 4 - right kidney; 5 - spleen; 6 - splenic vein; 7 - inferior mesenteric vein; 8 - left kidney.

Table 1. Vascular morphometry of the reported case

\begin{tabular}{lcc}
\hline Blood vessel & Calibre $[\mathrm{mm}]$ & Length $[\mathrm{cm}]$ \\
\hline Gastrosplenic trunk & 5.902 & 2.859 \\
Left gastric artery & 1.931 & \\
Splenic artery & 5.441 & \\
Hepatomesenteric trunk & 5.49 & 2.983 \\
Superior mesenteric artery & 6.66 & \\
Common hepatic artery & 4.401 & \\
Splenic vein & 9.729 & \\
Inferior mesenteric vein & 9.204 & \\
Superior mesenteric vein & 8.921 \\
Portal vein & 13.01 \\
\hline
\end{tabular}

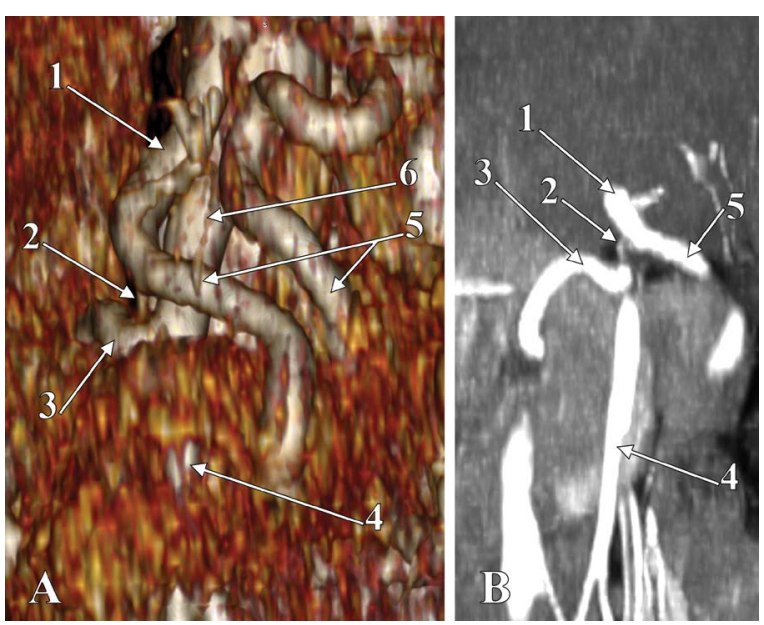

Figure 4. Three-dimensional volume renderisation (left antero-infero-lateral view, A), and multiplanar coronal slice (B) of the trunks emerging the upper segment of the abdominal aorta (AA). 1 - gastrosplenic trunk; 2 - arc of Bühler; 3 - common hepatic artery; 4 - superior mesenteric artery; 5 - splenic artery; 6 hepatomesenteric trunk. 
the GST in $4.1 \%$ of patients [31] but it was not found any GST associated with a HMT, such as in the present report. Uflacker (2006) [32] indicated that the GST might associate a middle hepatic artery origin from the aorta or the SMA. Wang et al. (2014) [33] listed two morphological possibilities in cases with HMT: HMT and GST, such as in this reported case, and HMT with distinct aortic origins for the LGA and SA.

The vertical insertion of the $C T$ in the aortic wall is variable, as related to vertebrae, being found as high as the T11-12 intervertebral disc level [34]. Different studies reporting GSTs did not mention the vertebral level of origin of that trunk, nor related it with the aortic hiatus of the diaphragm [12, 20]. Kahraman et al. (2001) [15] reported the origin of the GST just below the aortic hiatus. In the case reported here the GST emerged within, and not below, the aortic hiatus, which implies a degree of the GST compression during the contractions of the diaphragm. It should be considered here the median arcuate ligament syndrome in which a low insertion of the ligament or a high origin of arterial trunks may cause extrinsic compressions [23].

Different topographical patterns were indicated as possible for a CHA emerged from the HMT [11], all of them describing an exclusively ascending course of the CHA and its variable placement in relation with the hepatic portal vein and the pancreas. However, none of these could be fitted with our findings, as the hepatic portal vein was horizontal and the CHA looped around it.

Common hepatic artery could ascend either anterior $[8,12,14,16,20]$, or posterior $[12,20,22,28]$ of the PV. Higashi and Hirai (1995) [11] described four types of HMT morphology. In types I-III the course of the CHA was posterior to the PV but in type IV the $\mathrm{CHA}$ ascended behind the superior mesenteric vein, passed around in front from the right side of the PV, and reached the liver [10], as was found in our case.

Most HMTs were reported after anatomical dissections $[1,4,5,8,9,12,14-16,20,22,24,28]$, the computed tomographic evidence being scarce $[3,13,29,33]$.

A HMT could be described as a replaced CHA originating the SMA [7]. This variant corresponds to Michels' type 9 of hepatic artery, in which the author indicated that the coeliac hepatic artery is absent and the entire hepatic trunk derived from the SMA [19]. That variant was found by Michels (1966) [19] in 5 of 200 dissections. There is, however, an incomplete correspondence of our variant and Michels' type 9 of hepatic artery. This is because Michels indicated the origin of the GDA from the GST [19], while we found that the GDA left the CHA. Hicks et al. (2016) [10] indicated Michels' type 9 of hepatic arterial anatomy by a diagram in which the GDA leaves the LGA, and not the GST, as in Michels' original diagram (1966) [19]. The GDA origin from a replaced CHA, as in our case, corresponds to Tandler's type 5 of CT variation [30]. Therefore, Tandler's type 5 of hepatic artery and, respectively, Michels' type 9 of hepatic artery are reciprocally exclusive. Unlike in Tandler's type 5 we found here an arc of Bühler uniting the GST and HMT.

During development the segmental ventral branches of the abdominal aorta are united by longitudinal anastomoses that may either persist or disappear $[12,18,25,30]$. There are three known possibilities of anastomoses between the $\mathrm{CT}$ and the SMA, the direct one, well known and rarely reported, being the arc that Bühler described in $1904[2,17]$. We report here a variant of the Bühler's arc that unites the GST and HMT instead of the CT and the SMA. This variant was not reported previously, at least to our knowledge.

Michalinos et al. (2019) [18] reviewed the literature regarding the arc of Bühler and discussed that, although the arc of Bühler is regarded as a remnant of Tandler's ventral longitudinal anastomosis, its embryogenesis is related to that of the dorsal pancreatic artery. This is because that artery could either originate from the SA, or from the SMA, and when both origins are maintained, an arc of Bühler results [18]. This theory is supported by the present findings: an absent dorsal pancreatic artery, seemingly replaced morphologically by a short arc of Bühler.

\section{CONCLUSIONS}

Arterial variations in the coeliac region are accurately identified on computed tomography angiograms. They should be evaluated by surgeons when different surgical procedures are evaluated.

\section{Conflict of interest: None declared}

\section{REFERENCES}

1. Adachi B. Das arteriensystem der japaner: In 2 bdn: Maruzen Company; 1928.

2. Bergman RA, Tubbs RS, Shoja MM. Bergman's comprehensive encyclopedia of human anatomic variation. John Wiley \& Sons, Hoboken, New Jersey 2016. 
3. Bueschel P, Meyer F, Weber M, et al. Rare aneurysm of the hepatic artery with overlap to the gastroduodenal artery in very uncommon coincidence with occurence of hepatomesenteric trunk. Wien Klin Wochenschr. 2013; 125(3-4): 111-114, doi: 10.1007/s00508-012-0317-8, indexed in Pubmed: 23420527.

4. Chandramohan K, Abdulla FA, Thomas S. Periampullary carcinoma complicated by a transpancreatic hepatomesenteric trunk-a case report of an extremely rare vascular anomaly. Indian J Surg Oncol. 2020; 11(1): 142-146, doi: 10.1007/ s13193-019-01001-9, indexed in Pubmed: 32205984.

5. Chitra R. Clinically relevant variations of the coeliac trunk. Singapore Med J. 2010; 51(3): 216-219, indexed in Pubmed: 20428743.

6. Grigoriță L, Damen NS, Vaida MA, et al. Unusual anatomical variation: tetrafurcation of the celiac trunk. Surg Radiol Anat. 2019; 41(11): 1399-1403, doi: 10.1007/s00276-01902286-9, indexed in Pubmed: 31302730.

7. Ha HII, Kim MJ, Kim J, et al. Replaced common hepatic artery from the superior mesenteric artery: multidetector computed tomography (MDCT) classification focused on pancreatic penetration and the course of travel. Surg Radiol Anat. 2016; 38(6): 655-662, doi: 10.1007/s00276016-1618-9, indexed in Pubmed: 26758052.

8. Harada H, Yamaki K, Doi $Y$, et al. An anomalous case of the gastro-splenic and the hepato-mesenteric trunks independently arising from the abdominal aorta. Kurume Med J. 1996; 43(2): 181-184, doi: 10.2739/kurumemedj.43.181, indexed in Pubmed: 8755123.

9. Hemamalini A, Pushpalatha K. Absence of celiac trunk associated with a hepatomesenteric trunk: A case report. J Anat Soc India. 2017; 66: S94, doi: 10.1016/j. jasi.2017.08.296.

10. Hicks CW, Burkhart RA, Weiss MJ, et al. Management of type 9 hepatic arterial anatomy at the time of pancreaticoduodenectomy: considerations for preservation and reconstruction of a completely replaced common hepatic artery. J Gastrointest Surg. 2016; 20(7): 1400-1404, doi: 10.1007/ s11605-016-3154-7, indexed in Pubmed: 27138326.

11. Higashi N, Hirai K, Higashi N, et al. [On the hepatic artery arising from the superior mesenteric artery]. Kaibogaku Zasshi. 1995; 70(4): 338-346, indexed in Pubmed: 8540282.

12. Hirai $Y$, Yamaki $K$, Saga $T$, et al. Two anomalous cases of the hepato-mesenteric and the gastro-splenic trunks independently arising from the abdominal aorta. Kurume Med J. 2000; 47(3): 249-252, doi: 10.2739/kurumemedj.47.249, indexed in Pubmed: 11059229.

13. lacob N, Pureca A, Belic O, et al. A hepatomesenteric trunk, in association with left gastric and splenic arteries arising independently from the abdominal aorta: A case report using MDCT angiography. J Anat Soc India. 2014; 63(2): 179-182, doi: 10.1016/j.jasi.2014.07.001.

14. limura A, Oguchi T, Shibata M, et al. An anomalous case of the hepatic artery arising from the superior mesenteric artery. Okajimas Folia Anat Jpn. 2007; 84(2): 61-65, doi: 10.2535/ofaj.84.61, indexed in Pubmed: 17969994.

15. Kahraman G, Marur T, Tanyeli E, et al. Hepatomesenteric trunk. Surg Radiol Anat. 2001; 23(6): 433-435, doi: 10.1007/ s00276-001-0433-z, indexed in Pubmed: 11963627.

16. Kardile PB, Ughade JM, Ughade MN, et al. Anomalous origin of the hepatic artery from the hepatomesenteric trunk. J Clin Diagn Res. 2013; 7(2): 386-388, doi: 10.7860/ JCDR/2013/5304.2778, indexed in Pubmed: 23543854.

17. McNulty JG, Hickey N, Khosa F, et al. Surgical and radiological significance of variants of Bühler's anastomotic artery: a report of three cases. Surg Radiol Anat. 2001; 23(4): 277-280, doi: 10.1007/s00276-001-0277-6, indexed in Pubmed: 11694975.
18. Michalinos A, Schizas D, Ntourakis D, et al. Arc of Bühler: the surgical significance of a rare anatomical variation. Surg Radiol Anat. 2019; 41(5): 575-581, doi: 10.1007/ s00276-018-2168-0, indexed in Pubmed: 30552487.

19. Michels NA. Newer anatomy of the liver and its variant blood supply and collateral circulation. Am J Surg. 1966; 112(3): 337-347, doi: 10.1016/0002-9610(66)90201-7, indexed in Pubmed: 5917302.

20. Nakamura Y, Miyaki T, Hayashi S, et al. Three cases of the gastrosplenic and the hepatomesenteric trunks. Okajimas Folia Anat Jpn. 2003; 80(4): 71-76, doi: 10.2535/ ofaj.80.71, indexed in Pubmed: 14964466.

21. Nakano H, Kikuchi K, Seta Si, et al. A patient undergoing pancreaticoduodenectomy in whom involved common hepatic artery anomalously arising from the superior mesenteric artery was removed and reconstructed. Hepatogastroenterology. 2005; 52(66): 1883-1885, indexed in Pubmed: 16334799.

22. Nayak S. Hepatomesenteric trunk and gastro-splenicophrenic trunk. Int J Anat Variat. 2008; 1: 2-3.

23. Ozbülbül NI. CT angiography of the celiac trunk: anatomy, variants and pathologic findings. Diagn Interv Radiol. 2011; 17(2): 150-157, doi: 10.4261/1305-3825.DIR.328310.1, indexed in Pubmed: 20690078.

24. Rammohan A, Sathyanesan J, Palaniappan R, et al. Transpancreatic hepatomesenteric trunk complicating pancreaticoduodenectomy. JOP. 2013; 14(6): 649-652, doi: 10.6092/1590-8577/1641, indexed in Pubmed: 24216553.

25. Rusu MC, Jianu AM, Sztika D, et al. Three extremely rare anatomic variants of the hepatic artery. Ann Vasc Surg. 2011; 25(8): 1138.e1-1138.e7, doi: 10.1016/j.avsg.2011.03.011, indexed in Pubmed: 21680146.

26. Rusu MC, Manta BA. Novel anatomic variation: heptafurcation of the celiac trunk. Surg Radiol Anat. 2018; 40(4): 457-463, doi: 10.1007/s00276-018-1995-3, indexed in Pubmed: 29497808.

27. Rusu MC, Măru N, Rădoi PM, et al. Trifurcated external carotid artery and complete gamma-loop of its maxillary branch. Surg Radiol Anat. 2019; 41(2): 231-234, doi: 10.1007/ s00276-018-2142-x, indexed in Pubmed: 30483866.

28. Saga T, Hirao T, Kitashima S, et al. An anomalous case of the left gastric artery, the splenic artery and hepato-mesenteric trunk independently arising from the abdominal aorta. Kurume Med J. 2005; 52(1-2): 49-52, doi: 10.2739/ kurumemedj.52.49, indexed in Pubmed: 16119612.

29. Schwarz L, Huet E, Yzet T, et al. An extremely uncommon variant of left hepatic artery arising from the superior mesenteric artery. Surg Radiol Anat. 2014; 36(1): 91-94, doi: 10.1007/ s00276-013-1131-3, indexed in Pubmed: 23652481.

30. Tandler J. über die Varietäten der Arteria coeliaca und deren Entwickelung. Beiträge und Referate zur Anatomie und Entwickelungsgeschichte. 1904; 25(2): 473-500, doi: 10.1007/bf02300762.

31. Torres K, Staśkiewicz G, Denisow M, et al. Anatomical variations of the coeliac trunk in the homogeneous Polish population. Folia Morphol. 2015; 74(1): 93-99, doi: 10.5603/FM.2014.0059, indexed in Pubmed: 25792402.

32. Uflacker R. Atlas of vascular anatomy: An angiographic approach. Wolters Kluwer Health 2006.

33. Wang Yi, Cheng C, Wang Lu, et al. Anatomical variations in the origins of the celiac axis and the superior mesenteric artery: MDCT angiographic findings and their probable embryological mechanisms. Eur Radiol. 2014; 24(8): 1777-1784, doi: 10.1007/s00330-014-3215-9, indexed in Pubmed: 24859597.

34. Yang IY, Oraee S, Viejo C, et al. Computed tomography celiac trunk topography relating to celiac plexus block. Reg Anesth Pain Med. 2011; 36(1): 21-25, doi: 10.1097/ AAP.0b013e318203067f, indexed in Pubmed: 21455084. 